\title{
Effect of Deep Vibration on Physical State of Soil Being Changed
}

\author{
Vladimir D. Geidt ${ }^{1, *}$, Larisa V. Geidt ${ }^{1}$, Andrey V. Geidt ${ }^{1}$, Svetlana V. Sheshukova ${ }^{2}$ \\ ${ }^{1}$ Department of Construction Production, Industrial University of Tyumen, Tyumen, Russia \\ ${ }^{2}$ Department of Intercultural Communication, Industrial University of Tyumen, Tyumen, Russia
}

Received December 17, 2020; Revised October 7, 2021; Accepted October 21, 2021

\begin{abstract}
Cite This Paper in the following Citation Styles
(a): [1] Vladimir D. Geidt, Larisa V. Geidt, Andrey V. Geidt, Svetlana V. Sheshukova, "Effect of Deep Vibration on Physical State of Soil Being Changed," Civil Engineering and Architecture, Vol. 9, No. 7, pp. 2273 - 2277, 2021. DOI: 10.13189/cea.2021.090714.
\end{abstract}

(b): Vladimir D. Geidt, Larisa V. Geidt, Andrey V. Geidt, Svetlana V. Sheshukova (2021). Effect of Deep Vibration on Physical State of Soil Being Changed. Civil Engineering and Architecture, 9(7), 2273 - 2277. DOI: 10.13189/cea.2021.090714.

Copyright $\odot 2021$ by authors, all rights reserved. Authors agree that this article remains permanently open access under the terms of the Creative Commons Attribution License 4.0 International License

\begin{abstract}
The effect of vibration on soil compaction has gained a good reputation when consolidating an artificially improved foundation. The studies aimed at analyzing the effect of the deep vibration on the properties of the compacted water-saturated soil have made it possible to establish that vibration can be an effective means of consolidation speeding-up. Vibration contributes to the emergence of additional excess pressures in the pore water; the filtration rate due to the increased pressure gradient increases as well. Consolidation speeding-up (soil compaction by squeezing out the pore water) is quite relevant. It is clear that consolidation speeding-up of foundation water-saturated soil is extremely important in site preparation for reducing site preparation time up to a period of a short summer. The study focuses on the mechanism of deep vibration (longitudinal mechanical waves) having an effect on water-saturated soil consolidation. The mechanism is based on the changed physical state of the medium and takes into account its structure. Two soil models have been proposed. The first one-parameter soil model is called a "sandwich (layercake)". The second two-parameter soil model is called a "loose sandwich". In here, the first model is a special case of the second one. Both parameters have simple physical significance. The study shows that according to the proposed mechanism, the effect of vibration on soil consolidation is reduced to redistribution of the relationship between the stress in soil skeleton and the pore pressure versus the value of "vibration pressure" in such a
\end{abstract}

way that the pore pressure increases, nevertheless, cannot exceed the value of the external static load. The paper also presents the results of numerical calculations.

Keywords Water-Saturated Soil, Consolidation, Pore Pressure, Deep Vibration, Harmonic Oscillations, Stresses

\section{Introduction}

In general, site preparation is carried out by hydraulic earth-moving or dumping using construction machinery. With a sufficiently large thickness of the foundation water-saturated soil and their low filtration capacity, stabilization is achieved in an unacceptably long period (several years). This factor is one of the most significant conditions that significantly increase the construction time. Therefore, consolidation speeding-up being introduced up to a period of a short summer (2-3 months) is economically sound in site preparation.

At present, it seems possible to consider research devoted to dynamic effects on soil consolidation to be accomplished (e.g. [1-5]). However, it can be stated with a certain degree of certainty that the mechanism of the vibration effect on soil consolidation has not been identified $[6,7]$. It is clear that the effect of deep vibration on soil consolidation is due to [7]:

occurrence of the additional alternative pressure; 
- changed filtration ability of the medium;

- changed physical state of the medium.

\section{Materials and Methods}

It has been actually proved in $[6,7]$ that the pore overpressure represented both by a harmonic law [6] and as a plane wave [7] does not leave "residual" effects in the medium after eliminating such an effect. It has been shown in [4] that the filtration coefficient does not depend on vibration. Therefore, the second of the above listed mechanisms disappears too. Let us consider the third of the above listed mechanisms of deep vibration on soil consolidation process.

Let the considered completely water-saturated soil (gas phase is absent) be affected by a static external load $\mathbf{q}$ directed vertically downward. A deep vibrator, which is a vertically mounted cylinder (radius $\mathbf{r}_{0}$ ), is turned on in this medium at $\mathrm{t}=0$; its "wall" creates harmonically changing perturbations in the medium with frequency $v$. It is clear that these perturbations propagate in the medium as mechanical waves.

It is known that mechanical waves can be surface, transverse and longitudinal (by the pattern of periodic motion of particles in the medium) (e.g. [8]). Surface waves are not discussed in the paper, since the authors consider deep vibration, and the upper part of the soil is externally loaded to a large extent. Surface waves occur at the interface between liquid and gas. It is known that transverse waves, (e.g. [8]) propagate only in solids. Transverse waves do not pass through liquids and bulky materials.

Let us consider interaction of longitudinal waves (propagating horizontally) with the medium. Taking into account the vibrator geometry, which is a source of longitudinal waves in the medium, we consider cylindrical longitudinal waves. For such waves, particle displacement versus rest position $\mathrm{u}=\mathrm{u}(\mathrm{r}, \mathrm{t})$ is described by the expression [8]

$$
u=\frac{a}{\sqrt{r}} \sin (\omega t-k r) e^{-\alpha r}
$$

where $a$ - value relating to the amplitude of oscillation: $u_{0}=a / \sqrt{r}$;

$\omega$ - wave phase frequency, $\omega=2 \pi v$,

where $v$ - oscillation frequency;

$k$-wave number $k=\omega / V$;

$V$ - wave velocity;

$r$ - distance from the axis of symmetry to the observation point;

$t$ - vibration time;

$\alpha$ - attenuation coefficient due to absorption of waves.

In here, additional pressure created by such waves is evaluated by the formula [6]:

$$
\widetilde{P}=\rho V \dot{u}=\rho V \omega u_{0} \cos (\omega t-k r) e^{-\alpha r},
$$

where $\rho$ - water density, $\alpha$ - attenuation coefficient.

The intensity of the wave $I(r)$ (average energy power over a period per unit of sectional area), taking into account attenuation, is

$$
I(r)=\frac{1}{2} \rho V \frac{\omega^{2} a^{2}}{r} e^{-2 \alpha r},
$$

Next, let us consider the effect of the wave on the state of water in the medium. To do this, let us start with an elementary act of interaction. Let the stress $\sigma$ occur in the soil skeleton. Each elementary particle of the soil is surrounded by a layer of water; this means that if to choose any 2 elementary particles (through which interaction is transferred), then the layer of water of thickness $\mathbf{b}$ will be between these particles. These particles are involved in the transfer of stress $\sigma$ in the soil skeleton. This means that the considered layer of bound water between particles behaves as a continuation of the skeleton. In the horizontal direction, this element is affected by the forces created by the wave, which are opposed by the friction forces.

Let us introduce the tangential stress $\tau$ created by the wave in the considered layer of water. It is clear that if $\tau$ does not exceed the shear stress created by the friction forces $\tau_{\mathrm{fr}}$, then the layer of water will be a single whole with soil particles. However, if $\tau$ exceeds $\tau_{\text {frr }}$, then this layer of water will move in relation to the skeleton and interaction between these two particles will break, and the water in this layer can be considered as free. Then these two particles will hang in the water, and the pressure $\sigma$ acting on the upper particle will not be transferred to the lower one, but will be transferred to the water. Thus, the upper particle taking the vertical pressure $\sigma$ will interact with water, i.e. pore pressure will increase by $\sigma$. Given the negligibly small compressibility of water, the process of water structure destruction between particles will occur without any change of the porosity coefficient $\mathbf{e}$ of the medium. Now let us assess $\tau$ and $\tau_{\text {fr }}$ quantitatively.

It is well known that the maximum value of $\tau_{\mathrm{fr}}$ is evaluated by the Coulomb formula in a macro volume (e.g. $[5,9])$ :

$$
\tau_{\mathrm{fr}}=\tau_{\text {Coul }}=\left(\sigma+\sigma_{\mathrm{c}}\right) \operatorname{tq} \varphi=\sigma \operatorname{tq} \varphi+\mathrm{C},
$$

where $\sigma$ - vertically directed load;

$$
\begin{aligned}
& \sigma_{\mathrm{s}} \text { - structural strength; } \\
& \mathrm{C} \text { - cohesion; } \\
& \varphi \text { - angle of internal friction. }
\end{aligned}
$$

Taking into account the principle of superposition, when macro properties are determined by the behavior of particles at the micro level, $\tau_{\mathrm{fr}}$ equals to $2 \tau_{\text {Coul }}$. Multiplier " 2 " is taken since both particles (the upper and the lower ones) participate in friction. 


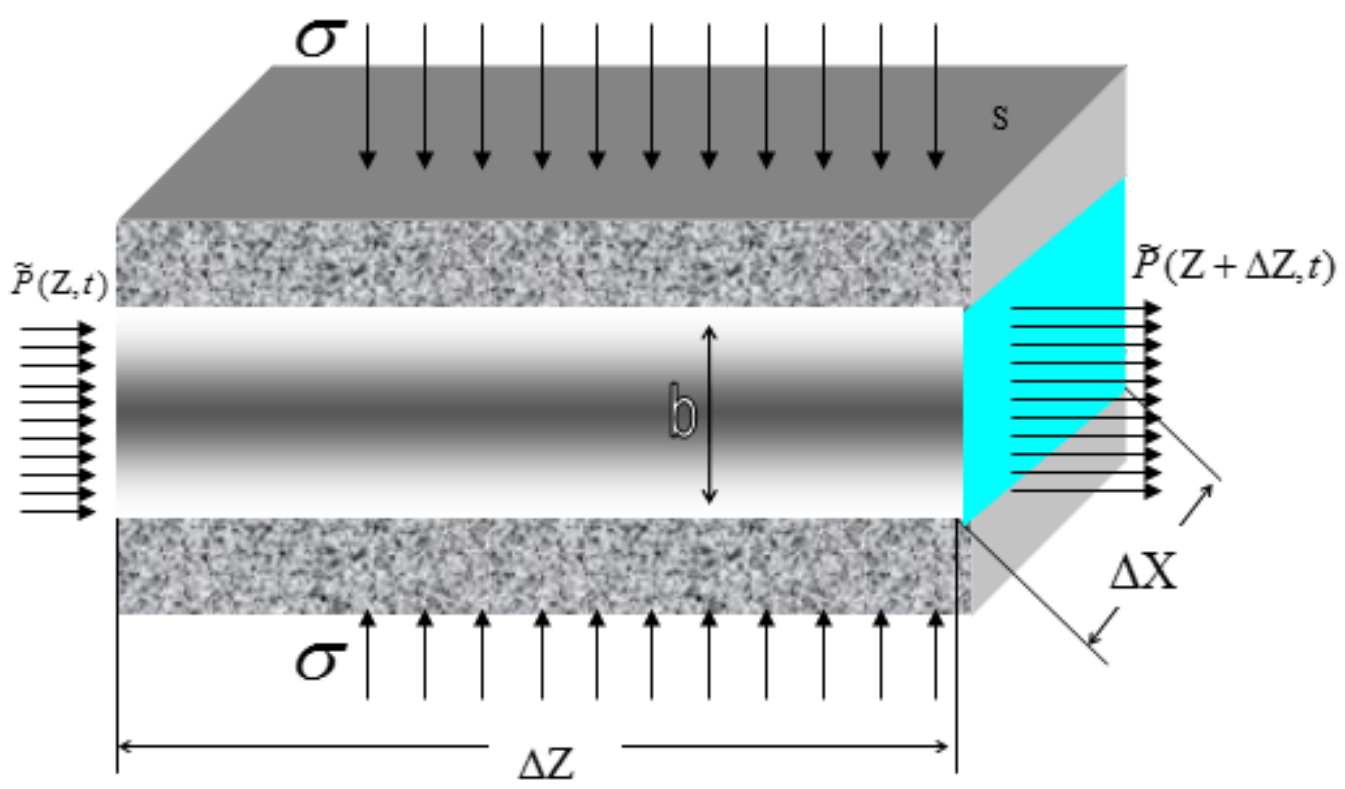

Figure 1. Schematic illustration of the effect of a mechanical plane wave on a layer of water enclosed between the selected particles ("flakes") of soil.

Now let us evaluate the shear stress $\tau \equiv \mathrm{F} / \mathrm{S}$ created by the plane wave in the selected layer of water located between the particles under consideration (see Figure 1).

Here: $\sigma$ - stresses in the soil skeleton; $b$ - distance between soil particles; $\widetilde{P}$ - wave overpressure at the given point at time $\mathrm{t} ; \mathrm{S}$ - area of soil part.

Thus, for the plane wave:

$$
\tau=\frac{F}{S}=\frac{\widetilde{P}_{z}^{\prime} b \Delta z \Delta x}{\Delta z \Delta x}=\widetilde{P}_{z}^{\prime} b
$$

Where $\widetilde{P}_{z}^{\prime}=d \widetilde{P} / d z$;

$\Delta z, \quad \Delta x$ - horizontal dimensions of the selected volume of soil;

$\mathrm{b}$ - average distance between particles.

Here and further, the $\sim$ sign above the letter $\widetilde{P}$ means the wave overpressure in contrast to the excess pore pressure $\mathbf{P}$.

In the case of a cylindrical wave (see Figure 2):

$$
\tau=\frac{F}{S}=b \frac{1}{r} \frac{\partial}{\partial r}[r \widetilde{P}(r, t)]
$$

Thus, the requirement to achieve complete liquefaction is as follows:

$$
\tau \geq \tau_{\text {Coul }}=\sigma \operatorname{tq} \varphi,
$$

where $\tau$ - tangential stress created by the wave in the considered layer of water; it is evaluated by the formulas (5) or (6). In here, (complete liquefaction), the pore pressure $\mathbf{P}$ reaches its maximum value $\mathbf{q}$.

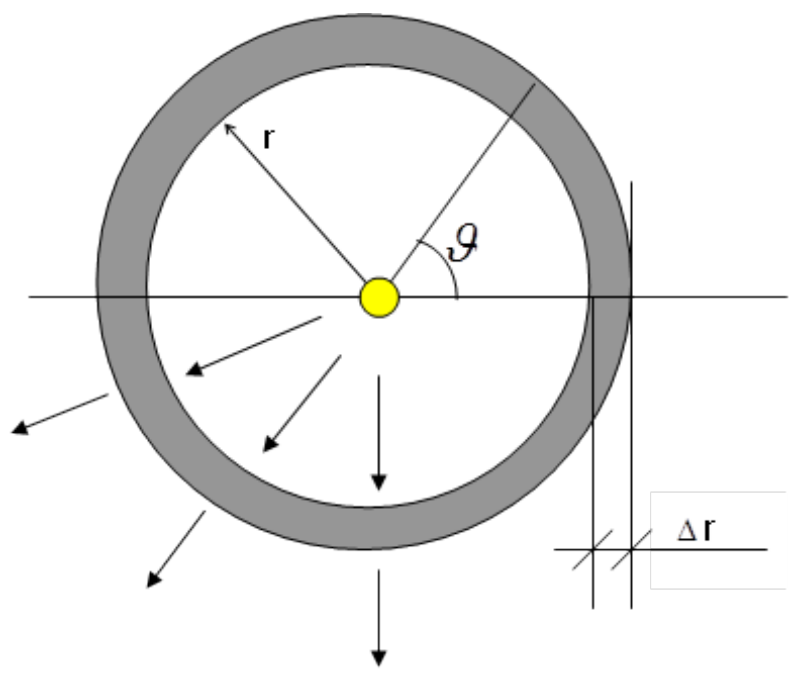

Figure 2. Schematic illustration of a cylindrical wave acting upon a layer of water enclosed between soil particles.

A source of cylindrical waves is shown in the center. Here $\vartheta$ - angle of the sector; $r$ - distance from the wave source to the layer under consideration; $\Delta \mathrm{r}$ - width of the element.

Next, let us consider the case when $\tau<\tau_{\text {Coul }}=\sigma$ tq $\varphi$. Taking into account that the transfer of stress $\sigma$ from the overlying soil layers to the underlying soil layers happens in such a way that some particles are tightly connected and some are weakly connected, i.e. when longitudinal waves act on such a medium, there will always be a pair of particles for which the wave action will be sufficient to break structural bonds. This means that structural bonds will not be broken for all particles, but only for some of 
them so that $\sigma$ decreases by $\Delta \sigma$ equal to

$$
\Delta \sigma=\frac{\tau}{2 \operatorname{tg} \varphi}
$$

In other words, the component $\Delta \sigma \mathrm{tq} \varphi$ equals to $\tau$ in the inequation $\tau<\tau_{\text {Coul }}=\sigma \operatorname{tq} \varphi=(\widetilde{\sigma}+\Delta \sigma) \operatorname{tq} \varphi$. In here, the stress in the skeleton $\sigma$ decreases by $\Delta \sigma$ and becomes equal to $\widetilde{\sigma}$ under the effect of the wave. Thus, the stress $\sigma$ in the skeleton in the absence of the wave is due to the residual stress in the skeleton in the presence of the wave $\widetilde{\sigma}$ by the relation $\widetilde{\sigma}=\sigma-\Delta \sigma$.

Taking into account that the stress in the skeleton both in the presence of the wave $\widetilde{\sigma}$ and the absence of the wave $\sigma$ is related to the pore pressure by the equation $\mathrm{q}=\sigma+\mathrm{P}$, we obtain that the pore pressure in the presence of the wave $\widetilde{P}$ does not reach the maximum value $\mathbf{q}$ under $\tau<\tau_{\text {Coul }}$. The state of the medium, when the pore pressure increases by $\Delta \sigma$ (i.e. $\widetilde{P}=P+\Delta \sigma$ ), but does not reach its maximum possible value $\mathbf{q}$, may be called partial liquefaction.

Thus, the effect of the wave on the medium under partial liquefaction $\left(\tau<\tau_{\text {Coul })}\right)$ implies that the pore pressure $\mathbf{P}$ increases as compared to $\widetilde{P}$ by $\Delta \sigma=\frac{\tau}{2 \operatorname{tg} \varphi}$, where $\tau$ is evaluated by the formula (5) for a plane wave and by (6) - for a cylindrical wave.

The evaluation formulas for $\tau$ can be obtained for the cylindrical wave, when the pore pressure changes according to the law

$$
P(r, t)=\rho v \omega \frac{a}{\sqrt{r}} \cos (\omega t-k z) e^{-\alpha r}
$$

Then:

$$
\begin{aligned}
& \tau=\frac{b}{r} \frac{\partial}{\partial r}[r P(r, t)]=\frac{b}{r} \rho V \omega a\left[\sqrt{r} e^{-\alpha r} \cos (\omega t-k z)\right]_{r}= \\
& =b \rho V \omega a \frac{1}{r \sqrt{r}} e^{-\alpha r}\left[\left(\frac{1}{2}-\alpha r\right) \cos (\omega t-b z)+k z \sin (\omega t-k z)\right]= \\
& =b \rho V \omega a \frac{1}{r \sqrt{r}} e^{-\alpha r} \Phi(\varphi)
\end{aligned}
$$

where $\Phi(\varphi)$ - value which depends on the wave phase $\varphi=$ $\omega \mathrm{t}-\mathrm{kr}$. When the period of oscillation $\mathrm{T}=1 / \nu=2 \pi / \omega$ is much less than the period of relaxation, $\Phi(\varphi)$ can be replaced with the maximum value of $\Phi_{\mathrm{m}}=\max \Phi(\varphi)(0 \leq$ $\mathrm{t} \leq 2 \pi)$, since if the friction forces are overcome between the particles in the layer of water at some value of the phase (at some point in time), then the structural bonds will not be capable to recover during oscillations in this layer of water. It can be illustrated as follows:

$$
\Phi_{m}=\frac{1}{2} \sqrt{(1-2 \alpha)^{2}+(2 k z)^{2}}=
$$

$$
=\frac{1}{2} \sqrt{1-4 \alpha r+4 r^{2}\left(\alpha^{2}+k^{2}\right)}
$$

Then the maximum value of $\tau_{m}(r) \equiv \max _{t_{1} \leq t \leq t_{1}+T} \tau(r, t)$ takes the form:

$$
\tau_{m}=b \rho V\left(\frac{\omega}{r}\right) \frac{a}{\sqrt{r}} \frac{1}{2} \sqrt{1-4 \alpha r+4 r^{2}\left(\alpha^{2}+k^{2}\right)}
$$

$\alpha$ - attenuation coefficient in these formulas.

Now let us reveal the relationship between the inter-pore gap $\mathbf{b}$ and the porosity coefficient $\mathbf{e}$. It is clear that this relationship depends upon soil structure. Therefore, b depends on the accepted ideas about soil structure. If to consider the soil model of "sandwich" type ("layer cake") which consists of dense flakes, $\delta$ in thickness and the distance between the flakes is $\mathbf{b}$, one can get:

$$
\mathrm{b}=\mathrm{e} \delta
$$

If to consider the soil model of "loose sandwich" with the inner porosity coefficient $\boldsymbol{e}_{\boldsymbol{u}}$ in its flakes, then

$$
b=\delta \frac{e-e_{u}}{1+e_{u}}
$$

If $e_{4}=0$ in the formula (13), then (13) goes over to the formula (12). Thus, the "flaky soil" model (12) is a special case of the "loose flaky sandwich" model (13).

For further discussion, let us restrict ourselves to the model (13), although it is clear that in order to cover different types of soils, the model (13) is not enough.

\section{Conclusions}

Proposed is the model for interaction of the longitudinal mechanical waves with water-saturated loaded soil. It has been revealed that:

- when the requirement (7) is fulfilled, the liquefaction state occurs (when the stress decreases to zero in the skeleton, i.e. $\widetilde{\sigma}=0$, and the pore pressure results in the increase to the maximum possible value $P=\mathrm{q}$ ); when $\tau_{\mathrm{m}}<\tau_{\text {Coul }}$, the stress decreases to the residual stress $\widetilde{\sigma}=\sigma-\Delta \sigma$ in the skeleton, but not to zero; thus, $\Delta \sigma$ can be evaluated by the formula (8) taking into account $\tau=\tau_{\mathrm{m}}$ where $\tau_{\mathrm{m}}$ is evaluated by the formula (6) for cylindrical waves where the parameter b is evaluated by the formula (13) for the "loose flaky sandwich" model. In the case under consideration $\tau_{\mathrm{m}}<$ $\tau_{\text {Coul }}$ (partial liquefaction), the pore pressure increases by $\Delta \sigma$ and does not reach the maximum possible value $\mathbf{q}$.

The studies have made it possible to conclude that vibration can be an effective means of consolidation speeding-up. It is clear that consolidation speeding-up of 
foundation water-saturated soil is extremely important in site preparation for reducing site preparation time up to a period of a short summer.

\section{REFERENCES}

[1] Konovalov P.A. Construction of Buildings in Peat Lands. Production and Practical Publication.-M.: Stroyizdat, 1995.-343 p. [rus]

[2] Armand A. Fondjo, Thywill C. Dzogbewu, "Swelling Stress and Suction Correlation of Compacted, Heaving Soils," Civil Engineering and Architecture, Vol. 8, No. 4, pp. 721 733, 2020. DOI: 10.13189/cea.2020.080434.

[3] Lusmeilia Afriani, Gatot Eko Susilo , Sri Nawangrini , Iswan, "Soil Shrinkage and Consolidation Study on Flood Embankments in Swamp irrigation Areas," Civil Engineering and Architecture, Vol. 8, No. 6, pp. 1225 1233, 2020. DOI: 10.13189/cea.2020.080607

[4] Kushnir S.Ya. Consolidation Phenomena in Peat Soils under Dynamic Impacts - In the book: Materials of the II Baltic Conference on Soil Mechanics and Foundation Engineering. Construction in Peat Lands and Deformations of Structures on Highly Compressible Soils.-Tallinn. 1988.- pp. 210-213. [rus]

[5] Ivanov P.L. Soils and Foundations of Hydraulic Structures. Soil Mechanics: Textbook for university students of hydraulic engineering specialties.-2nd ed., revised and ext.-M.: Higher school, 1991.- 447 p. [rus]

[6] Ufukov P.P., Geidt V.D., Geidt L.V. Effect of Extra Dynamic Load on Soil Consolidation Varying in Time according to Harmonic Law. // Manuscript dep. at VINITI, Moscow, 2000. [rus]

[7] Ufukov P.P., Geidt V.D., Ufukova O.Yu. Soil Consolidation Equation with Extra Dynamic Impact by Plane Wave. // Manuscript dep. at VINITI, Moscow, 2000. [rus]

[8] Koshkin N.I., Shishkevich M.G. Handbook on Elementary Physics. -8th ed., Revised.-M.: Nauka. Chief Ed. Board. Phys.-math. Lit., 1980.- 280 p. [rus]

[9] Abelev M.Yu. Residential and Industrial Construction on Weak Water-saturated Soils.- M.: Stroyizdat, 1983. - 248 p. [rus]

[10] Zaretsky Yu.K. Peat Foundation Consolidation // Foundations, Bases and Soil Mechanics. - 1970.- № 6- pp. 12-15. [rus] 